\title{
Prevalence of Access Recirculation in Prevalent Arterio-Venous (A-V) Fistula Hemodialysis Patients and Its Effect on Hemodialysis Adequacy
}

\author{
Michael Mamdouh Fakhry, Essam Nour El Din, Lina Khedr
}

Internal Medicine Department, Ain Shams University

Corresponding author: Michael Mamdouh Fakhry; Mobile: 0127007720; Email: mike.mamdouh95@ gmail.com

\begin{abstract}
Background: Assessment of access recirculation (AR) is crucial to dialysis efficiency and there is thus a need for a method yielding a highly accurate, fast, easy and economical measurement that can be applied in any dialysis clinic. Non-urea based dilutional methods are more accurate than urea based methods and avoid problems with cardiopulmonary recirculation, but they require expensive specialized devices, which limit their applicability. Patients and Methods: We used simple dilutional method of AR based on the determination of serum potassium $[\mathrm{K}+]$ in two samples. A prospective study was performed in a Dialysis Unit at El Sahel Teaching hospital, Cairo, on End stage kidney disease patients on regular Hemodialysis through a functioning Arterio-venous fistula. Results: Access recirculation was found in $42 \%$ of studied patients. There were Highly Significant positive correlation between access recirculation, pre / post dialysis blood urea, basal k, and parathyroid hormone level. In addition, there were highly significant negative correlation between AR, Urea reduction ratio and KT/V. Conclusion and Recommendations: Potassium dilution method is one of the most simple, specific, and economical way to measure access recirculation and can easily be performed in any dialysis unit. We recommend more research should be done about hemodialysis adequacy, access recirculation and how to improve it.
\end{abstract}

Keywords: Access, Recirculation, Hemodialysis, Potassium, Dilutional

\section{INTRODUCTION}

End stage renal disease (ESRD) is one of the most common life-threatening diseases. The number of patients accepted for renal replacement therapy including hemodialysis (HD), peritoneal dialysis, and kidney transplantation in developed and developing countries is increasing every year and imposes a major social and economic burden on these communities ${ }^{(1)}$.

Some factors that affect the survival of ESRD patients including inadequate dialysis, method of renal replacement therapy, etiology of renal failure and the presence of comorbid disease ${ }^{(2) .}$

It is well established that one of causes of inadequate dialysis in HD patients is arterio-venous (A-V) fistula access recirculation (AR). In addition, screening for recirculation may be used as a surveillance technique for the early detection of fistula stenosis, the correction of which may prevent thrombosis ${ }^{(3)}$.

Hemodialysis access recirculation is diagnosed when dialyzed blood returning through the venous side reenters the dialyzer through the arterial needle, rather than returning to the systemic circulation and as a result, the efficiency of HD is reduced $^{(4) \text {. }}$

The Diagnosis of arterio-venous (A-V) fistula access recirculation (AR) could be done through two methods Non Urea based method (Ultrasound dilution Transonic Hemodialysis Monitor-USM) or Dilution techniques, utilizing the dilution of serum potassium (K), glucose, hematocrit $(\mathrm{Hct})$ and hemoglobin $(\mathrm{Hb})$ have been recently described ${ }^{(5)}$.

Assessment of access recirculation (AR) is crucial to dialysis efficiency and there is thus a need for a method yielding a highly accurate, fast, easy and economical measurement that can be applied in any busy dialysis clinic. So the Non urea based methods are good method \& avoid problems with cardiopulmonary recirculation, but they require expensive specialized devices, which limit their applicability.

In Dilutional-based method, the degree of access recirculation is measured by comparing the basal and arterial $\mathrm{k}$ concentration from the following formula: Percent recirculation $=100 \times[1$ - arterial $\mathrm{K}^{+} /$basal $\mathrm{K}^{+}$.

Potassium-based dilutional method, similar to other dilutional methods, is not influenced by cardiopulmonary recirculation or veno-venous disequilibrium and is fast and accurate. Moreover it is very simple, economical, and can easily be performed in any dialysis unit ${ }^{(6)}$.

The potassium-based method showed: sensitivity $(100 \%)$; specificity $(95 \%)$; predictive value, positive $(91 \%)$; predictive value, negative $(100 \%)$. In addition, the potassium-based method appears to be more reliable than the two-needle urea based method ${ }^{(6)}$. 


\section{PATIENTS AND METHODS}

\section{Patients:}

This study was carried out in a Dialysis Unit at El Sahel Teaching Hospital, Cairo, from April to June, 2017. The study was approved by the Ethics Board of Ain Shams University.

Fifty patients were included in this study, $34(68 \%)$ males and

$16(32 \%)$ females, with ages ranging from 22 to 62 years old. All of them

were with ESKD on regular HD through a functioning AVF for at least 3 months duration.

Inclusion criteria:- patients older than 18 years old. End Stage Renal Disease patients on regular hemodialysis more than 3 months. Dialysis through arterio-venous $(\mathrm{A}-\mathrm{V})$ fistula

Exclusion criteria:- patients younger than 18 years old. ESRD patients on regular hemodialysis less than 3 months. Dialysis through tunneled catheter. Patients with A.V fistula those were nonfunctioning or malfunctioning by physical examination.

\section{Sampling}

At this thesis we used the dilution techniques, utilizing the dilution of serum potassium (K).

The degree of recirculation will be measured with potassium-based dilution method of $\mathrm{AR}$ which does not require any specific device, based on the determination of serum potassium $\left[\mathrm{K}^{+}\right]$in two samples.

A basal sample is drawn at the time of needle insertion (basal $\mathrm{K}^{+}$); needles are connected to blood lines and blood flow rate is quickly increased to $300 \mathrm{ml} / \mathrm{min}$.

A second sample (arterial $\mathrm{K}^{+}$) is drawn from the arterial line port within 5 to 10 seconds, to avoid errors due to cardiopulmonary recirculation of the normal saline entering the blood stream.

If there is no access recirculation, the basal $\mathrm{K}^{+}$level will be equal to the arterial $\mathrm{K}^{+}$level. Therefore, the percentage will be zero. However, if recirculation is present, part of the normal saline will enter the arterial line and dilute serum $\mathrm{K}^{+}$and the level of diluted arterial $\mathrm{K}^{+}$will determine the degree of $\mathrm{AR}$ according to the equation:
The AR formula is AR $(\%)=100 \times[1-$ arterial $\mathrm{K}^{+}$/basal K $\mathrm{K}^{+}$.

The access recirculation is considered positive if it was more than 0 percent but is considered significant for further investigation if it was more than 5 percent

The percentage of access recirculation will be related to the adequacy of AVF and dialysis through $\mathrm{kt} / \mathrm{v}$ as well as other routine investigation ${ }^{(7)}$.

\section{Ethical considerations:-}

Informed consent was taken from all participant patients.

\section{RESULTS}

Fifty patients were included in this study, $34(68 \%)$ males and 16 (32\%) females, with ages range from $22-62$ years old.

All of them were ESKD on regular HD through a functioning AVF. $15(30 \%)$ of them having distal, $35(70 \%)$ proximal, 39 (78\%) of patients were receiving dialysis through their first fistula and 11 (22\%) from second AVF and dialysis duration ranged from $10-197$ month. 26 (52.0\%) of them were $\mathrm{HCV}$ ab positive and the other 24 (48.0\%) patients were HCV ab negative.

Their dry weight in $\mathrm{kg}$ ranged from $45-94$ $\mathrm{kg}$, their systolic blood pressure ranged from $90-160$ $\mathrm{mmHg}$, their diastolic blood pressure ranged from 60 $-90 \mathrm{mmHg}$ and MAP ranging from $60-110 \mathrm{mmHg}$.

Pre-dialysis blood Urea level (urea 1) ranged from $79-237 \mathrm{mg} / \mathrm{dl}$ and post-dialysis blood urea level (urea 2) was ranged from $20-115 \mathrm{mg} / \mathrm{dl}$

Urea reduction ratio URR ranged from 37 $-78 \%$ And the KT/V ranged From $0.7-1.8$.

Access recirculation was found in $42 \%$.

We divided the patients into two groups' one group with an AVF access recirculation and the other did not have an AVF access recirculation.

We compared between the two groups regarding Age, dry wt in $\mathrm{kg}$, Avf position, duration of ESRD in months, $\mathrm{BP}$, arterial $\mathrm{K}$, alb, $\mathrm{Ca}$, phosphorus, $\mathrm{Hb}$, CRP, Cholesterol, LDL, HDL, TG $\&$ fasting glucose. There was highly significant positive correlation between access recirculation and urea1, urea2, basal $\mathrm{K}^{+}$, and PTH level. There was highly significant negative correlation between access recirculation and URR and KT/V. 
Our study did not find any relation between access recirculation and other parameters like $\mathrm{Hb}, \mathrm{Ca}$, lipid profile, age, sex or related to position of the fistula or its being the first or second time access.

Table (1): Different demographic data among all studied patients.

\begin{tabular}{|c|c|c|}
\hline \multicolumn{2}{|c|}{ Study patients } & No. $=50$ \\
\hline Age in years & $\begin{array}{l}\text { Mean } \pm \text { SD } \\
\text { Range }\end{array}$ & $\begin{array}{c}43.40 \pm 10.57 \\
22-62\end{array}$ \\
\hline Sex & $\begin{array}{l}\text { Female } \\
\text { Male }\end{array}$ & $\begin{array}{l}16(32.0 \%) \\
34(68.0 \%)\end{array}$ \\
\hline Dry weight in $\mathrm{kg}$ & $\begin{array}{l}\text { Mean } \pm \text { SD } \\
\text { Range }\end{array}$ & $\begin{array}{c}69.76 \pm 11.23 \\
45-94\end{array}$ \\
\hline duration of ESRD in months & $\begin{array}{l}\text { Mean } \pm \text { SD } \\
\text { Range }\end{array}$ & $\begin{array}{c}71.36 \pm 53.22 \\
10-197\end{array}$ \\
\hline \multirow{2}{*}{ AVF } & $\begin{array}{l}\text { Distal } \\
\text { Proximal }\end{array}$ & $\begin{array}{l}15(30.0 \%) \\
35(70.0 \%)\end{array}$ \\
\hline & $\begin{array}{l}\text { First one } \\
\text { Second one }\end{array}$ & $\begin{array}{l}39(78.0 \%) \\
11(22.0 \%)\end{array}$ \\
\hline Systolic BP & $\begin{array}{l}\text { Mean } \pm \text { SD } \\
\text { Range }\end{array}$ & $\begin{array}{c}123.40 \pm 14.23 \\
90-160\end{array}$ \\
\hline Diastolic BP & $\begin{array}{l}\text { Mean } \pm \text { SD } \\
\text { Range }\end{array}$ & $\begin{array}{c}79.80 \pm 8.69 \\
60-90\end{array}$ \\
\hline MAP & $\begin{array}{l}\text { Mean } \pm \text { SD } \\
\text { Range }\end{array}$ & $\begin{array}{c}86.42 \pm 10.12 \\
60-110\end{array}$ \\
\hline $\mathrm{HCV} \mathrm{Ab}$ & $\begin{array}{l}\text { Negative } \\
\text { Positive }\end{array}$ & $\begin{array}{l}24(48.0 \%) \\
26(52.0 \%)\end{array}$ \\
\hline & $\begin{array}{l}\text { Sex } \\
\text { Sivile al Wuk }\end{array}$ & \\
\hline
\end{tabular}

Figure (1): Percentage distributions of HD patients according to gender.

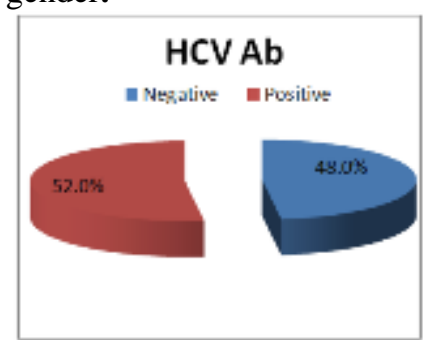

Figure (2): Percentage distributions of HD patients according to $\mathrm{HCV}$ ab.

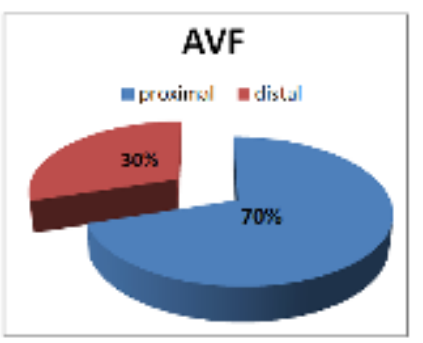

Figure (3): Percentage distributions of HD patients according to AVF Position.
Table (2): Blood Urea, URR \& KT/V of the studied patients.

\begin{tabular}{|l|l|c|}
\hline \multicolumn{2}{|c|}{} & No. $=\mathbf{5 0}$ \\
\hline Blood UREA 1(pre -dialysis & Mean \pm SD & $144.84 \pm$ \\
urea level) (in mg/dl) & Range & 32.77 \\
$79-237$ \\
\hline \multirow{2}{*}{ Blood UREA 2(post -dialysis } & Mean \pm SD & $56.88 \pm$ \\
urea level) ((in mg/dl) & Range & 21.43 \\
& Mean \pm SD & $60.90 \pm 9.79$ \\
URR\% & Range & $37-78$ \\
\hline \multirow{2}{*}{ K T $\backslash V$} & Mean \pm SD & $1.19 \pm 0.26$ \\
& Range & $0.7-1.8$ \\
\hline
\end{tabular}

Table (3): Basal K, Arterial K \& access recirculation of the studied patients.

\begin{tabular}{|l|l|c|}
\hline \multirow{2}{*}{ Basal K (in meq/l) } & Mean \pm SD & $5.24 \pm 0.79$ \\
& Range & $3.9-6.9$ \\
\hline \multirow{2}{*}{ Arterial K (in meq/l) } & Mean \pm SD & $4.69 \pm 0.61$ \\
& Range & $3.7-6.9$ \\
\hline \multirow{2}{*}{ Access Recirculation } & Mean \pm SD & $10.26 \pm 7.29$ \\
& Range & $>0-34.7$ \\
\hline
\end{tabular}

Table (4): The Prevalence of Access Recirculation in studied patients.

\begin{tabular}{|l|c|c|}
\hline \multicolumn{1}{|c|}{ Access Recirculation } & NO. & \% \\
\hline No Access recirculation & 29 & $58.0 \%$ \\
Access recirculation & 21 & $42.0 \%$ \\
\hline
\end{tabular}

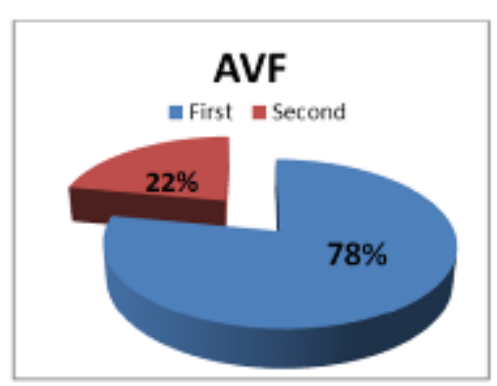

Figure (4): Percentage distributions of HD patients according to the number of AVF done.

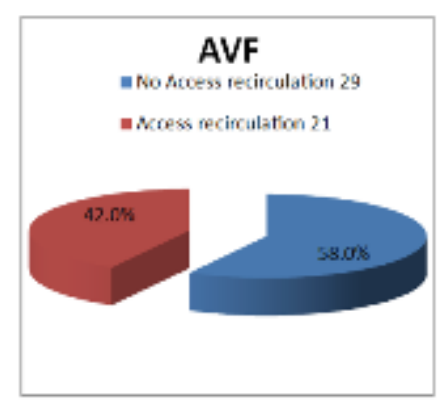

Figure (5): Prevalence of Access Recirculation . 
Table (5): Comparison between patients with no access recirculation and those with access recirculation regarding demographic data like Age, sex, dry wt in $\mathrm{kg}$, Avf position, duration of ESRD in months, BP \& HCVab.

\begin{tabular}{|c|c|c|c|c|c|}
\hline & & \multirow{2}{*}{\begin{tabular}{|c|}
$\begin{array}{c}\text { No Access } \\
\text { recirculation }\end{array}$ \\
No. $=29$ \\
\end{tabular}} & \multirow{2}{*}{\begin{tabular}{|c|}
$\begin{array}{c}\text { Access } \\
\text { recirculation }\end{array}$ \\
No. $=21$ \\
\end{tabular}} & \multicolumn{2}{|c|}{$\begin{array}{c}\text { Independent } \\
\text { t-test }\end{array}$} \\
\hline & & & & $\mathbf{t} / \mathbf{X}^{2 *}$ & P-value \\
\hline Age in years & $\begin{array}{l}\text { Mean } \pm \text { SD } \\
\text { Range } \\
\end{array}$ & \begin{tabular}{|c|}
$44.03 \pm 11.66$ \\
$22-62$
\end{tabular} & $\begin{array}{c}42.52 \pm 9.04 \\
28-56 \\
\end{array}$ & 0.495 & 0.623 \\
\hline Sex & \begin{tabular}{|l|} 
female \\
male
\end{tabular} & \begin{tabular}{|c|}
$8(27.6 \%)$ \\
$21(72.4 \%)$ \\
\end{tabular} & $\begin{array}{c}8(38.1 \%) \\
13(61.9 \%) \\
\end{array}$ & $0.618 *$ & 0.432 \\
\hline Dry weight in $\mathrm{kg}$ & $\begin{array}{l}\text { Mean } \pm \text { SD } \\
\text { Range }\end{array}$ & $\begin{array}{c}70.59 \pm 12.26 \\
45-94\end{array}$ & $\begin{array}{c}68.62 \pm 9.81 \\
53-91\end{array}$ & 0.607 & 0.547 \\
\hline \multirow{2}{*}{ Position } & \begin{tabular}{|l} 
Distal \\
Proximal \\
\end{tabular} & $\begin{array}{c}7(24.2 \%) \\
22(75.8 \%) \\
\end{array}$ & $\begin{array}{c}8(38.1 \%) \\
13(61.9 \%) \\
\end{array}$ & $0.618 *$ & 0.432 \\
\hline & \begin{tabular}{|l|} 
First one \\
Second one
\end{tabular} & \begin{tabular}{|c|}
$25(86.2 \%)$ \\
$4(13.8 \%)$ \\
\end{tabular} & \begin{tabular}{|c|}
$14(66.7 \%)$ \\
$7(33.3 \%)$ \\
\end{tabular} & $0.002 *$ & 0.963 \\
\hline $\begin{array}{l}\text { duration of ESRD } \\
\text { in months }\end{array}$ & \begin{tabular}{|l|} 
Range \\
Range \\
\end{tabular} & \begin{tabular}{|c|}
$71.59 \pm 53.16$ \\
$10-195$ \\
\end{tabular} & \begin{tabular}{|c|}
$71.05 \pm 54.61$ \\
$14-197$ \\
\end{tabular} & 0.035 & 0.972 \\
\hline systolic & $\begin{array}{l}\text { Mean } \pm \text { SD } \\
\text { Range }\end{array}$ & $\begin{array}{c}122.07 \pm 15.90 \\
90-160 \\
\end{array}$ & \begin{tabular}{|c|}
$125.24 \pm 11.67$ \\
$110-140$ \\
\end{tabular} & -0.774 & 0.443 \\
\hline diastolic & $\begin{array}{l}\text { Mean } \pm \text { SD } \\
\text { Range }\end{array}$ & \begin{tabular}{|c|}
$78.28 \pm 9.28$ \\
$60-90$ \\
\end{tabular} & \begin{tabular}{|c|}
$81.90 \pm 7.50$ \\
$70-90$ \\
\end{tabular} & -1.475 & 0.147 \\
\hline MAP & $\begin{array}{l}\text { Mean } \pm \text { SD } \\
\text { Range }\end{array}$ & $\begin{array}{c}85.10 \pm 11.20 \\
60-110 \\
\end{array}$ & $\begin{array}{c}88.24 \pm 8.34 \\
80-100 \\
\end{array}$ & -1.083 & 0.284 \\
\hline $\mathrm{HCV} \mathrm{Ab}$ & $\begin{array}{l}\text { Negative } \\
\text { Positive }\end{array}$ & \begin{tabular}{|l|}
$14(48.3 \%)$ \\
$15(51.7 \%)$ \\
\end{tabular} & $\begin{array}{l}10(47.6 \%) \\
11(52.4 \%)\end{array}$ & $0.002 *$ & 0.963 \\
\hline
\end{tabular}

*:Chi-square test

$P>0.05$ : Non significant

$P<0.05$ : Significant

$\mathrm{P}<0.01$ : Highly significant

Our study found that there were no significant relations between access recirculation and all the previous demographic data like Age, sex, dry wt in $\mathrm{kg}$, Avf position, duration of ESRD in months, BP \& HCVab.

Table (6): Comparison between patients with no access recirculation and those with access recirculation regarding $\mathrm{HD}$ adequacy parameters like urea, URR, KT/V, \& k level.

\begin{tabular}{|c|c|c|c|c|c|}
\hline & \multirow{2}{*}{\begin{tabular}{|c|}
$\begin{array}{c}\text { No Access } \\
\text { recirculation }\end{array}$ \\
No. $=29$ \\
\end{tabular}} & \multirow{2}{*}{\begin{tabular}{|c|}
$\begin{array}{c}\text { Access } \\
\text { recirculation }\end{array}$ \\
No. $=21$ \\
\end{tabular}} & \multicolumn{2}{|c|}{$\begin{array}{c}\text { Independent } \\
\text { t-test }\end{array}$} \\
\hline & & & & $t$ & P-value \\
\hline $\begin{array}{l}\text { UREA 1(pre-dialysis } \\
\text { urea level) }\end{array}$ & \begin{tabular}{|l}
$\begin{array}{l}\text { Mean } \pm \text { SD } \\
\text { Range }\end{array}$ \\
\end{tabular} & $\begin{array}{c}135.17 \pm 24.35 \\
92-190 \\
\end{array}$ & $\begin{array}{c}158.19 \pm 38.44 \\
79-237 \\
\end{array}$ & -2.591 & 0.013 \\
\hline $\begin{array}{l}\text { UREA 2(post--dialysis } \\
\text { urea level) }\end{array}$ & $\begin{array}{l}\text { Mean } \pm \text { SD } \\
\text { Range }\end{array}$ & $\begin{array}{c}4.83 \pm 11.84 \\
20-70 \\
\end{array}$ & $\begin{array}{c}73.52 \pm 20.67 \\
35-115 \\
\end{array}$ & -6.213 & 0.001 \\
\hline URR \% & $\begin{array}{l}\text { Mean } \pm \text { SD } \\
\text { Range }\end{array}$ & \begin{tabular}{|c|}
$66.36 \pm 7.46$ \\
$40-78$ \\
\end{tabular} & $\begin{array}{c}53.35 \pm 7.33 \\
37-64 \\
\end{array}$ & 6.132 & 0.001 \\
\hline $\mathrm{K} T \backslash \mathrm{V}$ & $\begin{array}{l}\text { Mean } \pm \text { SD } \\
\text { Range }\end{array}$ & $\begin{array}{c}1.35 \pm 0.20 \\
1-1.8\end{array}$ & $\begin{array}{c}0.97 \pm 0.13 \\
0.7-1.2\end{array}$ & 7.604 & 0.001 \\
\hline Basal K & \begin{tabular}{|l|}
$\begin{array}{l}\text { Mean } \pm \text { SD } \\
\text { Range }\end{array}$ \\
\end{tabular} & $\begin{array}{c}4.93 \pm 0.68 \\
3.9-6.9 \\
\end{array}$ & $\begin{array}{c}5.66 \pm 0.75 \\
4.4-6.9\end{array}$ & -3.560 & 0.001 \\
\hline Arterial K & $\begin{array}{l}\text { Mean } \pm \text { SD } \\
\text { Range }\end{array}$ & $\begin{array}{c}4.70 \pm 0.71 \\
3.7-6.9\end{array}$ & $\begin{array}{c}4.68 \pm 0.47 \\
3.9-5.3\end{array}$ & 0.115 & 0.909 \\
\hline
\end{tabular}

Our study found that there was no relation between access recirculation and arterial $\mathrm{K}$. However, there was highly significant relations between access recirculation and UREA 1, UREA 2, URR\%, K T / V \& Basal K.

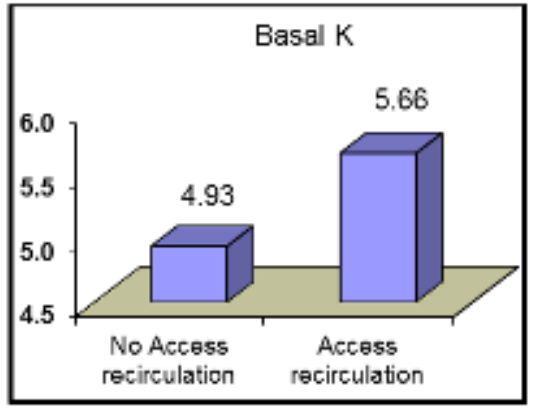

Figure (6): Comparison between patients with no access recirculation and those with access recirculation regarding Basal $\mathrm{K}$ level.

We found that the mean level of basal $\mathrm{K}$ among patients with access recirculation was higher than the mean basal $\mathrm{k}$ level in patient having no access recirculation.

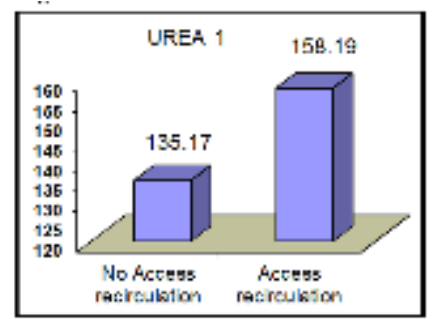

Figure (7): Comparison between patients with no access recirculation and those with access recirculation regarding pre-dialysis blood urea level (urea 1).

We found that the mean level of predialysis blood urea among the patients with access recirculation was higher than the mean of predialysis blood urea level in patients having no access recirculation.

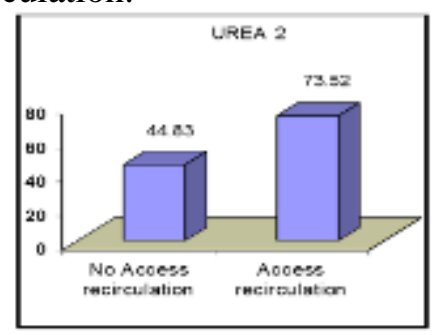

Figure (8): Comparison between patients with no access recirculation and those with access recirculation regarding post-dialysis blood urea level (urea 2).

We found that the mean level of postdialysis blood urea among the patients with access recirculation was higher than the mean of postdialysis blood urea level in patients having no access recirculation. 


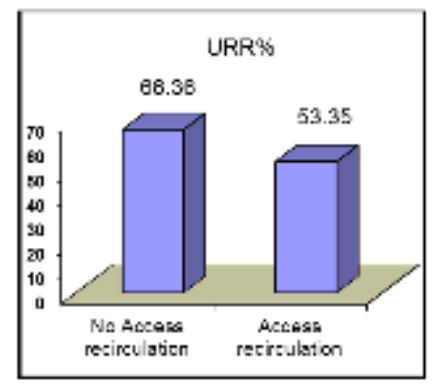

Figure (9): Comparison between patients with no access recirculation and those with access recirculation regarding urea Reduction ratio.

We found that the mean level of urea reduction ratio among the patients with access recirculation was lower than the mean of urea reduction ratio among patients having no access recirculation.

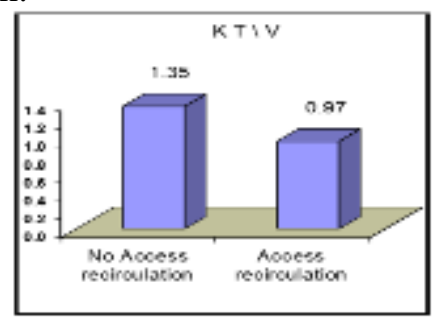

Figure (10): Comparison between patients with no access recirculation and those with access recirculation regarding $\mathrm{KT} / \mathrm{V}$.

The mean level of KT/V among the patients with access recirculation was lower than the mean of KT/V among the patients having no access recirculation.

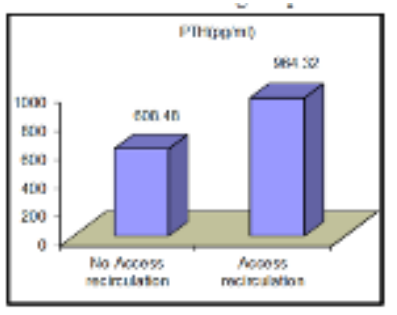

Figure (11): Comparison between patients with no access recirculation and those with access recirculation regarding PTH Level.

The mean level of PTH among the patients with access recirculation was higher than the mean of PTH among the patients having no access recirculation.
Table (8): showing a Comparison between patients with no access recirculation and those with access recirculation regarding other chemical data like alb, $\mathrm{Ca}, \mathrm{PO}_{4}, \mathrm{PTH}, \mathrm{Hb}, \mathrm{CRP}$, Cholest, LDL, HDL, TG \& Fasting Glucose.

\begin{tabular}{|c|c|c|c|c|c|}
\hline & & \multirow{2}{*}{$\begin{array}{c}\begin{array}{c}\text { No Access } \\
\text { recirculation }\end{array} \\
\text { No. }=29\end{array}$} & \multirow{2}{*}{$\begin{array}{c}\begin{array}{c}\text { Access } \\
\text { recirculation }\end{array} \\
\text { No.=21 } \\
\end{array}$} & \multicolumn{2}{|c|}{$\begin{array}{c}\text { Independent } \mathrm{t} \text { - } \\
\text { test }\end{array}$} \\
\hline & & & & $\mathbf{t}$ & $\begin{array}{c}\text { P- } \\
\text { value }\end{array}$ \\
\hline Serum po $4(\mathrm{mg} / \mathrm{dl})$ & $\begin{array}{l}\text { Mean } \pm \text { SD } \\
\text { Range }\end{array}$ & $\begin{array}{c}4.78 \pm 1.17 \\
2.7-7.5 \\
\end{array}$ & $\begin{array}{c}5.01 \pm 1.36 \\
2.4-7.2 \\
\end{array}$ & -0.643 & 0.523 \\
\hline $\begin{array}{l}\text { Serum } \\
\text { PTH(pg/ml) }\end{array}$ & \begin{tabular}{|l|} 
Mean \pm SD \\
Range
\end{tabular} & $\begin{array}{c}608.48 \pm 433.06 \\
225-1900\end{array}$ & \begin{tabular}{|c|}
$964.32 \pm 499.73$ \\
$75-1900$ \\
\end{tabular} & -2.688 & 0.010 \\
\hline $\mathrm{Hb}(\mathrm{gm} / \mathrm{dl})$ & \begin{tabular}{|l|} 
Mean \pm SD \\
Range
\end{tabular} & $\begin{array}{c}10.73 \pm 0.91 \\
9.2-12.8\end{array}$ & $\begin{array}{c}10.36 \pm 1.13 \\
8.6-12.7 \\
\end{array}$ & 1.298 & 0.200 \\
\hline $\begin{array}{l}\text { Cholesrtrol } \\
(\mathrm{mg} / \mathrm{dl})\end{array}$ & \begin{tabular}{|l|} 
Mean \pm SD \\
Range
\end{tabular} & $\begin{array}{c}171.59 \pm 38.90 \\
89-259 \\
\end{array}$ & $\begin{array}{c}158.24 \pm 51.08 \\
89-292 \\
\end{array}$ & 1.050 & 0.299 \\
\hline $\mathrm{TG}(\mathrm{mg} / \mathrm{dl})$ & \begin{tabular}{|l} 
Mean \pm SD \\
Range
\end{tabular} & $\begin{array}{c}174.45 \pm 94.94 \\
60-513 \\
\end{array}$ & $\begin{array}{c}165.52 \pm 54.07 \\
42-260 \\
\end{array}$ & 0.387 & 0.700 \\
\hline $\mathrm{HDL}(\mathrm{mg} / \mathrm{dl})$ & $\begin{array}{l}\text { Mean } \pm \text { SD } \\
\text { Range }\end{array}$ & $\begin{array}{c}44.03 \pm 8.32 \\
29-56\end{array}$ & $\begin{array}{c}43.24 \pm 9.01 \\
28-59\end{array}$ & 0.323 & 0.748 \\
\hline LDL(mg/dl) & $\begin{array}{l}\text { Mean } \pm \text { SD } \\
\text { Range }\end{array}$ & $\begin{array}{c}87.69 \pm 23.79 \\
50-145 \\
\end{array}$ & $\begin{array}{c}79.38 \pm 29.46 \\
41-135 \\
\end{array}$ & 1.102 & 0.276 \\
\hline $\begin{array}{l}\text { Fasting blood } \\
\text { glucose }(\mathrm{mg} / \mathrm{dl})\end{array}$ & $\begin{array}{l}\text { Mean } \pm \text { SD } \\
\text { Range }\end{array}$ & $\begin{array}{c}94.17 \pm 6.84 \\
82-108\end{array}$ & $\begin{array}{c}96.90 \pm 7.89 \\
83-107\end{array}$ & -1.307 & 0.197 \\
\hline
\end{tabular}

Our study found that there was no relation between access recirculation and alb, $\mathrm{Ca}, \mathrm{PO} 4, \mathrm{Hb}$, CRP, Cholesterol, LDL, HDL, TG \& fasting glucose. However, there was highly significant relation between access recirculation and PTH level

Table (9): Correlation between access recirculation level and the other studied parameters like Age, sex, dry wt in $\mathrm{kg}$, Avf position, duration of ESRD in months, Bp \& HCV ab, Urea, URR, KT/V, K, alb, Ca, po4, PTH, Hb, CRP, Cholest, \& Fasting Glucose.

\begin{tabular}{|l|c|c|}
\hline \multirow{2}{*}{} & \multicolumn{2}{|c|}{ Access Recirculation } \\
\cline { 2 - 3 } & r & P-value \\
\hline Age in years & -0.034 & 0.815 \\
\hline Dry weight in kg & -0.067 & 0.642 \\
\hline duration of ESRD in months & 0.034 & 0.813 \\
\hline Systolic b.p. & 0.018 & 0.903 \\
\hline Diastolic b.p. & 0.135 & 0.351 \\
\hline MAP & 0.093 & 0.520 \\
\hline UREA 1 (pre-dialysis blood urea) & $0.367^{* *}$ & $\mathbf{0 . 0 0 9}$ \\
\hline UREA 2 (post-dialysis blood urea) & $0.786^{* *}$ & $\mathbf{0 . 0 0 1}$ \\
\hline URR\% & $-0.781^{* * *}$ & $\mathbf{0 . 0 0 1}$ \\
\hline K T $\backslash$ V & $-0.755^{* * *}$ & $\mathbf{0 . 0 0 1}$ \\
\hline Basal K & $0.572^{* *}$ & $\mathbf{0 . 0 0 1}$ \\
\hline Arterial K & -0.088 & 0.542 \\
\hline Serum albumin $(\mathrm{g} / \mathrm{dl})$ & -0.032 & 0.823 \\
\hline Serum Ca $(\mathrm{mg} / \mathrm{dl})$ & -0.182 & 0.210 \\
\hline Serum po4(mg/dl) & 0.082 & 0.572 \\
\hline Serum PTH(pg/ml) & $0.517^{* *}$ & 0.000 \\
\hline CRP & 0.211 & 0.141 \\
\hline Hb(mg/dl) & -0.113 & 0.433 \\
\hline Cholesrtrol(mg/dl) & -0.012 & 0.935 \\
\hline Fasting blood glucose(mg/dl) & 0.107 & 0.458 \\
\hline & & \\
\hline & &
\end{tabular}


There was no correlation between access recirculation and age, dry wt in $\mathrm{kg}$, Avf position, duration of ESRD in months, $\mathrm{BP}$, arterial $\mathrm{K}$, alb, $\mathrm{Ca}$, PO4, Hb, CRP, Cholesterol, LDL, HDL, TG\& fasting glucose. However, there was highly significant correlation between access recirculation and, Urea1\& 2, URR, KT/V, basal K, PTH Level.

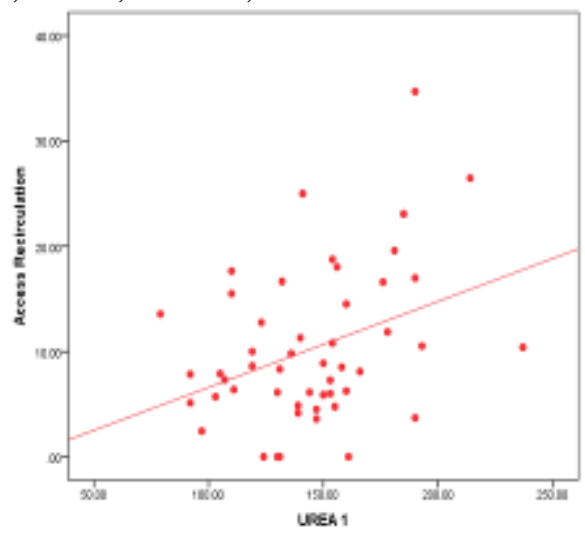

Figure (12): Correlation between access recirculation level and pre-dialysis blood urea.

There was positive correlation between access recirculation and pre-dialysis blood urea (urea1).

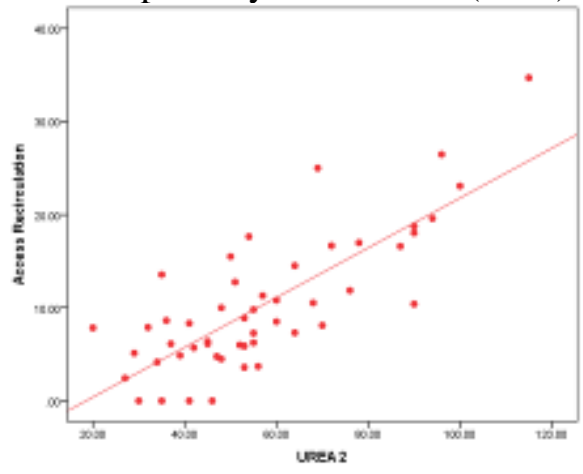

Figure (13): Correlation between access recirculation level and post-dialysis blood urea.

There was positive correlation between access recirculation and post-dialysis blood urea (urea2).

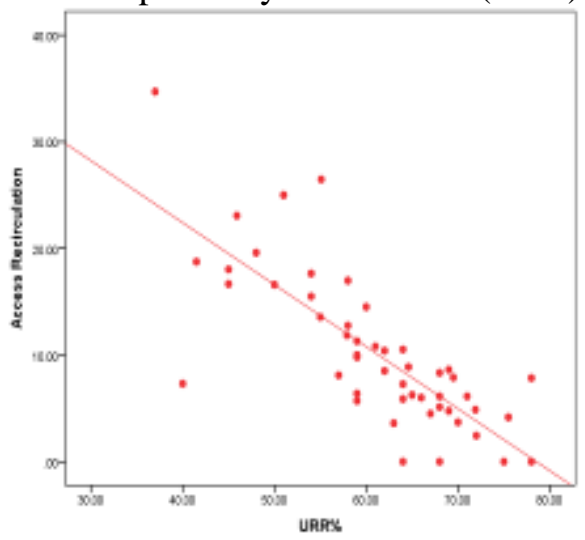

Figure (14): Correlation between access recirculation level and urea reduction ratio (URR\%).
There was negative correlation between access recirculation and urea reduction ratio (URR\%).

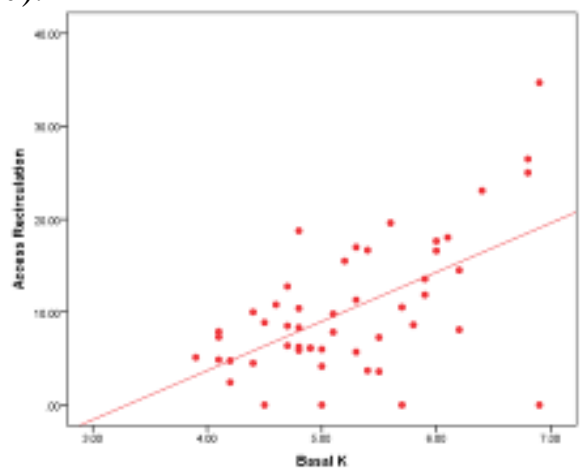

Figure (15): Correlation between access recirculation level and Basal K level.

There was positive correlation between access recirculation and basal $\mathrm{K}$ level.

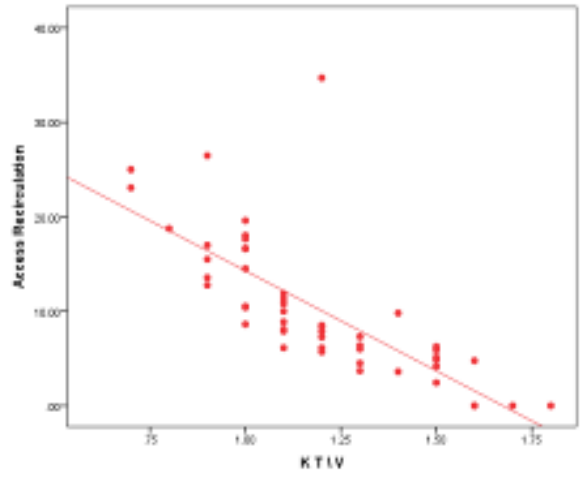

Figure (16): Correlation between access recirculation level and $\mathrm{KT} / \mathrm{V}$.

There was negative correlation between access recirculation and KT/V.

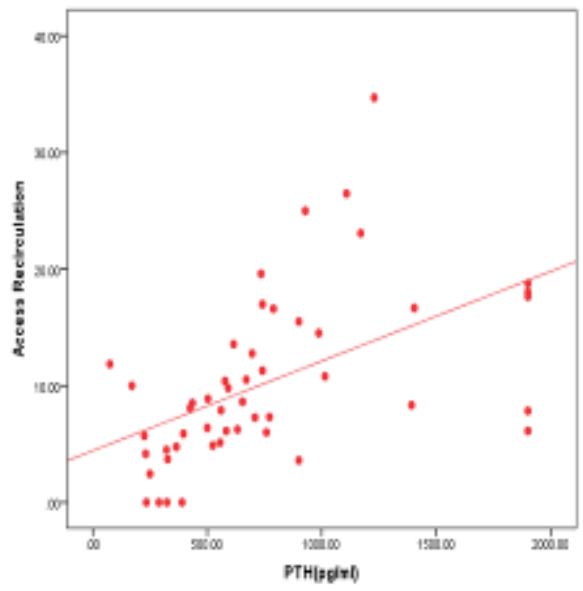

Figure (17): Correlation between access recirculation level and PTH level.

There was positive correlation between access recirculation and PTH level. 


\section{DISCUSSION \& CONCLUSION}

Hemodialysis constitutes the most common form of renal replacement therapy (RRT) worldwide. The goal of dialysis in patients with ESKD is to restore body's extracellular and intracellular composition to that of normal to the greatest extent possible and the surrogate marker for this physiological achievement of dialysis in clinical practice is the measurement of "adequacy of dialysis". Inadequate dialysis is responsible for the high mortality of patients with ESKD. Apart from duration of dialysis and blood flow rate, the body surface area of the patient, composition of diet, nutritional status, etc. may influence the adequacy of dialysis ${ }^{(8)}$.

It was found that urea reduction ratio (URR) and $\mathrm{Kt} / \mathrm{V}$, are good indicators of dialysis adequacy and URR greater than $65 \%$ and a $\mathrm{Kt} / \mathrm{V}$ greater than 1.2 are recommended for adequate HD. Each 0.1 decrease in $\mathrm{Kt} / \mathrm{V}$ is associated with approximately $7 \%$ increase in the relative risk of death and $11 \%$ increase in the annual rates of hospitalization ${ }^{(9)}$.

It is well established that one of causes of inadequate dialysis in HD patients is arterio-venous (A-V) fistula access recirculation (AR) . In addition, screening for recirculation may be used as a surveillance technique for the early detection of fistula stenosis, the correction of which may prevent thrombosis ${ }^{(3)}$.

This study is an epidemiological study to investigate the prevalence of arterio-venous $(\mathrm{A}-\mathrm{V})$ fistula access recirculation (AR) and its effect on adequacy of hemodialysis in patients using AVF as a vascular access.

The result showed that from all study patient only 21 patients have access recirculation and their access recirculation ranged from $>0-$ $34.7 \%$ making the prevalence of access recirculation in our study was $42.0 \%$. Hayati et al. (10) found that the Access recirculation in their study only $17 \%$ of patients and their study was on 100 hemodialysis (HD) patients with arteriovenous $(\mathrm{A}-\mathrm{V})$ fistula who were on HD for more than 3 months by using urea based method .

As regard to urea reduction ration (URR) And KT/V, the current study showed that URR and $\mathrm{Kt} / \mathrm{V}$ were decreased in patients group with access recirculation in a significant relation, which agreed with Coyne et al. ${ }^{(11)}$ Hemodialysis adequacy work group of 146 stable dialysis patients in whom measurements of $\mathrm{Kt} / \mathrm{V}$ and access recirculation were obtained every month for three successive months, 25 percent of low $\mathrm{Kt} / \mathrm{V}$ values resulted from significant access recirculation

As regards the increase in the pre- and post-dialysis blood urea level and level of basal potassium, they showed significant relation as their $\mathrm{p}$ value for pre dialysis blood urea level (urea 1) was 0.013 and 0.000 for post-dialysis blood urea level (urea 2) and for basal K was 0.001 that agreement with Stolic et al. ${ }^{(12)}$ They showed that there were significant increase in the levels of serum urea before and after session, as the group of patients who had adequate HD doses, had low level of urea before and after session.

This study showed that $15(30 \%)$ of patients had distal AVF and $35(70 \%)$ had proximal. From other view 39 (78\%) of them first fistula, $11(22 \%)$ second time shunt and there was no significant relation between that and access recirculation, which was in agreement with Mahbub et al. ${ }^{(13)}$ where radiocephalic was found $19(90.5 \%)$ in group I, 66 (79.5\%) in group II and 9 $(64.3 \%)$ in group III. Brachiocephalic was found 2 $(9.5 \%)$ in group I, $17(20.5 \%)$ in group II and $5(35.7 \%) \mathrm{n}$ group III. The difference was not statistically significant $(p>0.05)$ among the three groups in chi square test.

AS regards age and gender, the current study showed that $34(68 \%)$ males and $16(32 \%)$ females, with ages ranged from $22-62$ years old, mean age was $43.40 \pm 10.57$. There was no significant relation between age and gender and AVF access recirculation, which agreed with Raiesifar et al. ${ }^{(14)}$ who studied dialysis adequacy in patients of Abodan HD center. They showed that there was no statistically significant relationship between the dialysis and gender. In addition, Mahbub et al. ${ }^{(13)}$ reported that most of the patients the mean age was found $43.81 \pm 11.95$ years in group I and $43.58 \pm 12.89$ years in group II and $44.36 \pm 11.45$ years in group III. The mean age difference was not statistically significant $(p>0.05)$ among the three groups

As regard to pre and post dialysis blood pressure, there were no statistically significant relationship between blood pressure and HD adequacy among our studied patients. This agreed with Tayyebi et al. ${ }^{(15)}$ who showed that there was no significant relationship between blood pressure and dialysis adequacy, access recirculation, $\mathrm{Kt} / \mathrm{V}$, and URR. The mean pre- and post-dialysis blood 
pressure was 135.76 and $125.70 \mathrm{mmHg}$ respectively that was within the normal ranges according to the definitions of blood pressure.

As regards serum albumin, there was no statistically significant relationship with access recirculation. The study showed that no change in the levels of serum albumin associated with increase in AVF AR, which was in contrast with Stolic et al. (12) who showed that there was significant differences between levels of serum albumin, as the group of patients who had adequate HD doses, had high levels of serum albumin. While, the patients having access recirculation must had decrease in dialysis adequacy.

In regard to the level of PTH we found that there was significantly higher PTH level in patient with access recirculation with $\mathrm{p}$ value for PTH reached 0.010 .

In regard to all of virology state, lipid profile, blood glucose, serum calcium, phosphorus, and calcium phosphorus product, serum sodium levels there were no statistically significant relationships between them and Access recirculation.

\section{REFERENCES}

1. USRDS (2003): Overall hospitalization and mortality. American Journal of Kidney Disease, 42 (6-5): 136-140.

2. Goodkin D, Bragg-Gresham J, Koenig $\mathbf{K}$ et al. (2003): Association of comorbid conditions and mortality in hemodialysis patients in Europe, Japan, and the United States: the Dialysis Outcomes and Practice Patterns Study (DOPPS). Journal of the American Society of Nephrology, 14(12): 3270-3277.

3. Miskulin D, Meyer K and Martin A (2003): Comorbidity and its change predict survival in incident dialysis patients. American journal of kidney diseases, 41(1):149-161.

4. Berkoben $M$ and Blankestijn $P$ (2011): Arteriovenous fistula recirculation in hemodialysis. nephro-urology monthly, 19(2):1312-1318.

5. Basile $\mathbf{C}$, Ruggieri $\mathbf{G}$, Vernaglione $\mathbf{L}$ et al. (2003): A comparison of methods for the measurement of hemodialysis access recirculation. Journal of nephrology, 16 (6): 908-913.

6. Brancaccio D, Tessitore $\mathbf{N}$, Carpani $\mathbf{P}$ et al. (2001): Potassium-based dilutional method to measure hemodialysis access recirculation. The
International journal of artificial organs, 24(9): 606-613.

7. Gammaro L, Brancaccio $\mathbf{D}$, Tessitore $\mathbf{N}$ et al (2001): Potassium-based dilutional method to measure hemodialysis access recirculation. The International journal of artificial organs, 24(9):606-613.

8. Sultania $P$, Acharya $P$ and Sharma S (2009): Adequacy of hemodialysis in Nepalese patients undergoing maintenance hemodialysis. Journal of Nepal Medical Association, 48(173): 3-10.

9. Amini M, Aghighi M, Masoudkabir F et al. (2011): Hemodialysis adequacy and treatment in Iranian patients. A National Multicenter Study. International Journal of Kidney disease, 5 (2): 103-109.

10. Hayati F, Shayanpour $S$ and Faramarzi $M$ (2015): Access Recirculation in Hemodialysis. Shiraz E-Medical Journal, 16(3): e59875.

11. Coyne D, Delmez J, Spence G et al. (1997): Impaired delivery of hemodialysis prescriptions: an analysis of causes and an approach to evaluation. Journal of the American Society of Nephrology, 8(8): 13151318.

12. Stolic R, Trajkovic G, Stolic D et al. (2010): Nutrition parameters as HD adequacy markers. Hippokratia, 14(3) 193-197.

13. Mahbub T, Chowdhur M, Jahan F et al. (2015). Estimation of Recirculation in ArterioVenous Fistula among Haemodialysis Patients. Bangladesh Journal of Medicine,25(1): 17-20.

14. Raiesifar A, Torabpour M, Mohsenized $P$ et al. (2009): Dialysis adequacy inpatients of Abadan hemodialysis center. Iranian Journal of critical care nursing, 2 (3): 87- 90.

1. Tayyebi A, Eynollahi $B$ and Sherne $M$ (2012): The relationship between blood pressure and dialysis adequacy in dialysis patients. Iranian Journal of critical care nursing, 5(1): 49- 52. 